\title{
Pharmacologic Treatments for Irritable Bowel Syndrome: an Umbrella Systematic Review
}

\author{
Min Chen ${ }^{1}$, Tai-Chun Tang ${ }^{1}$, Di Qin ${ }^{2}$, Ling Yue ${ }^{2}$, Hui Zheng ${ }^{2}$
}

1) Colorectal Department/

Clinical Medicine College, Hospital of Chengdu University of Traditional Chinese Medicine, Chendu 2) The Third hospital/ Acupuncture and Tuina School, Chengdu University of Traditional Chinese Medicine, Chengdu, China

\footnotetext{
Address for correspondence: Hui Zheng

Chengdu University of Traditional Chinese Medicine, 1166 Liutai Avenue, Wenjiang District, Chengdu, 610000, China zhenghui@cdutcm.edu.cn
}

Received: 07.02.2020 Accepted: 13.05.2020

\begin{abstract}
Background \& Aims: Multiple pharmacologic treatments are available for the management of irritable bowel syndrome (IBS), and a large body of evidence has been presented. However, the strength and credibility of the evidence have not been comprehensively evaluated. We aimed to review the systematic reviews and metaanalyses of pharmacologic treatments for IBS and evaluate the credibility of the findings.

Methods: We searched MEDLINE, Embase, and Cochrane library from inception to September 2019 for systematic reviews evaluating the effectiveness of pharmacologic treatments for IBS. We summarized relative ratios (RR), evaluated the credibility of the evidence and classified the evidence into convincing, highly suggestive, suggestive, and weak.

Results: We included 11 systematic reviews with 40 meta-analyses (330 randomized controlled trials and 86,459 participants) assessing 10 treatment categories and 2 drugs. Most of the pharmacologic treatments were significantly superior over placebo as reported by the included meta-analyses. The evidence for 5-hydroxytryptamine (5-HT) 3 antagonists ( $\mathrm{RR}=1.56$, 95\% CI: 1.43-1.71), antispasmodics (RR=1.19, 95\%CI: 1.02-1.39), and alosetron ( $R R=1.46,95 \% \mathrm{CI}: 1.26-1.71)$ were highly suggestive for relieving global IBS symptoms. 5 -HT4 agonists ( $\mathrm{RR}=1.26,95 \% \mathrm{CI}: 1.19-1.34)$ and guanylate cyclase-C (GCC) agonists ( $\mathrm{RR}=1.73$, 95\%CI: 1.54-1.95) were found to give convincing evidence for the improvement of the responder rate. 5-HT3 antagonists (RR=1.32, 95\%CI: 1.26-1.38) offered convincing evidence for relieving abdominal pain.

Conclusions: Evidence for 5-HT3 antagonists, 5-HT4 agonists and GCC agonists, antispasmodics, and alosetron were suggestive for the treatment of IBS. However, owing to the risk of bias in randomization methods, the results for GCC should be interpreted with caution.
\end{abstract}

Key words: pharmacologic treatments - irritable bowel syndrome - umbrella review - meta-analysis.

Abbreviations: CI: confidence interval; E: expected number of randomized control trails with positive findings; ES: effect size; GCC: guanylate cyclase-C; IBS: irritable bowel syndrome; O: observed number of randomized control trails with positive findings; PI: prediction interval; RCT: randomized controlled trial; 5-HT: 5-hydroxytryptamine.

\section{INTRODUCTION}

Irritable bowel syndrome (IBS) is a functional bowel disorder characterized by abdominal pain or discomfort and a change in bowel habits in the absence of structural abnormalities. This disease is highly prevalent in the general population with a prevalence of $5-18 \%[1,2]$; it negatively affects working productivity and quality of life [3]. Patients with IBS have claimed to give up 10-15 years of life expectancy for an instant cure [4], which indicates its substantial impact on the quality of life. Direct costs associated with IBS management have been estimated at more than $\$ 1$ billion in the United states [5], and a meta-analysis with data from 23 European countries showed a mean annual cost of $€ 1837$ spent for treating IBS [6]. The heavy burden of IBS inspires research enthusiasm on developing treatment modalities.

Pharmacologic treatments are the primary choice in the management of IBS, especially when dietary interventions or life-style modification fail to relieve IBS symptoms $[4,7,8]$. Plenty of pharmacologic treatments are developed for IBS, since the pathology of IBS is not fully elucidated and response to treatment varies across IBS subtypes [4, 9]. A large body of research on the effectiveness of pharmacological treatments 
for IBS is available. Many published systematic reviews and meta-analyses summarized evidence on 5-hydroxytryptamine (5-HT)3 antagonists [10], 5-HT4 agonists [10], alosetron [11], antidepressants [12], antibiotics [13], antispasmodics [14], bulking agents [14], intestinal secretagogues [15], probiotics [13], prokinetics [14], and tegaserod [16]. The findings of these studies help to guide clinical practice in the treatment of IBS. However, the strength, precision, and potential bias of the findings remain unclear. Further summarizing the findings and evaluating their credibility could facilitate clinical decision making, and therefore are attractive for physicians and patients with IBS.

The umbrella systematic review is a recently developed method that allows a higher-level synthesis of current evidence and a better recognition of the uncertainties, potential bias, and knowledge gaps in the evidence [17]. It summarizes the findings of recently published systematic reviews and meta-analyses, and quantitatively evaluates the impact of potential bias (heterogeneity, publication bias, and excess significance bias) on the current evidence. Several umbrella reviews have been recently published to address key clinical questions such as the association between diet and diabetes [18], the impact of environmental risk on inflammatory bowel diseases [19], and the effect of dietary and nutritional interventions on cardiovascular outcomes [20], but no umbrella review has been reported to address the effect of pharmacologic treatments on IBS. We aimed to conduct an umbrella systematic review to obtain a comprehensive overview of the existing evidence of randomized controlled trials (RCTs) on pharmacologic treatments for IBS and to evaluate its strength and credibility.

\section{METHODS}

The design and implementation of this study were in accordance with the Preferred Reporting Items for Systematic Reviews and Meta-Analysis (PRISMA) statement [21] and with the methodological guide for conducting umbrella reviews [22]. The protocol was registered on PROSPERO (CRD42018109597) prior to the conduction of the study and was published elsewhere [23].

\section{Search strategy}

We searched MEDLINE (via OVID database), Embase and the Cochrane library for systematic reviews and metaanalyses that examined the effect of pharmacologic treatments on IBS from inception to September 2019. We developed a comprehensive search strategy incorporating the following terms: irritable bowel syndrome, systematic review, metaanalysis. No language restriction was set during the search. The search strategy for each database is provided in the Supplement file. We read the reference lists of the retrieved articles to search for additional systematic reviews, and we also searched medical journal websites (www.nejm.org, https://jamanetwork. com, www.thelancet.com, www.bmj.com, www.gastrojournal. org, and https://journals.lww.com/ajg/pages/default.aspx), government websites (www.fda.gov, www.nice.org.uk, www. sign.ac.uk, www.ahrq.gov), and PROSPERO for any missed systematic reviews.

\section{Study selection}

The inclusion criteria were: (1) systematic reviews that assessed the efficacy of pharmacologic treatments [4] (including 5-HT3 antagonists, 5-HT4 agonists, alosetron, antidepressants, antibiotics, antispasmodics, bulking agents, intestinal secretagogues, probiotics, prokinetics, and tegaserod) in adult participants with IBS (age $\geq 18$ years); (2) systematic reviews that included RCTs; (3) reporting outcomes of interests that included the assessment of global IBS symptoms, responder rate (a responder was defined as each meta-analysis described), abdominal pain or discomfort, defecation urgency, stool frequency, stool consistency, quality of life, and adverse events; (4) being compared with placebo or active control.

Systematic reviews with any of the following conditions were excluded: (1) without meta-analysis; (2) the number of included RCTs were less than 10 [24]; (3) full-text copy unavailable or without sufficient data for analysis; (4) inclusion of observational studies.

When multiple meta-analyses focusing on the same treatment and the same outcome measures were found, we selected the one with the largest number of participants and the most recent version. Two reviewers (T.-C.T. and D.Q.) independently screened the titles and abstracts of retrieved meta-analyses. Discrepancy was solved by group discussion and arbitrated by a third reviewer (H.Z). We examined the interrater reliability in the selection process by using kappa statistics.

\section{Data extraction and quality assessment}

Two reviewers (M.C. and L.Y.) independently extracted data from each meta-analysis. The extracted data included name of first author, year of publication, journal source, treatments, comparators, number of trials, total sample size of each meta-analysis, method used for pooling estimates (fixed or random effects), detection of publication bias, evaluation of heterogeneity, and assessment of risk of bias. Additionally, we extracted data from each original RCT on the name of RCT, first author, year of publication, treatment, outcomes, raw parameters (number of events, means, and standard deviations), and sample size for each treatment. A third reviewer (H.Z.) checked the completeness and correctness of the extracted data. The two reviewers also evaluated the quality of the included meta-analyses by using A MeaSurement Tool to Assess Systematic Reviews 2nd version (AMSTAR 2) [25]. We used AMSTAR 2 to evaluate 16 aspects of a meta-analysis. The authors of AMSTAR2 discouraged the use of a summary score for a meta-analysis to indicate its methodological quality, so we generated a summary figure to present the overall quality of current evidence (Supplement file Fig. 1).

\section{Data analysis}

We summarized the findings of each meta-analysis, and we re-calculated the summary effect size (ES) and its corresponding $95 \%$ confidence interval (CI) by using a random-effects model (meta package in $\mathrm{R}$ 3.5.0). We also estimated the $95 \%$ prediction interval (95\%PI) for each metaanalysis to assess whether they excluded null value; the 95\%PI accounts for heterogeneity between RCTs and specifies the uncertainty for the ES that would be expected in future studies. 
We assessed heterogeneity in each meta-analysis by using $\mathrm{I}^{2}$ statistics, and $\mathrm{I}^{2} \geq 50 \%$ was recognized as the sign of significant heterogeneity in a meta-analysis. We further categorized the degree of heterogeneity into small $\left(\mathrm{I}^{2}<25 \%\right)$, moderate $\left(25 \%<\mathrm{I}^{2}<50 \%\right)$, large $\left(50 \% \leq \mathrm{I}^{2}<75 \%\right)$, and significantly large $\left(\mathrm{I}^{2} \geq 75 \%\right)$. Publication bias and small-study effect were evaluated by examination of the symmetry of the funnel plot; significant publication bias was detected by using Egger's test, and $\mathrm{p}<0.01$ indicates existence of publication bias. We assessed the risk of excess significance bias in each meta-analysis by comparing the observed number of RCTs with positive findings (O) with the expected number (E). The E was estimated by the sum of study power that each RCT actually reached. We used the ES of the largest study as the true ES in estimating the study power, since the true ES of a treatment is unable to acquire [26]. We also calculated the ratio of $\mathrm{O}$ versus $\mathrm{E}$ to estimate the degree of excess significance bias.

We evaluated the credibility of current evidence by the following criteria [20, 24, 27]: (1) had $p<0.05$ in fixed-effects model or $\mathrm{p}<0.001$ in random-effects model; (2) had the total sample size larger than 1000; (3) had 95\%PI that excluded the null value; (4) had no significant heterogeneity $\left(\mathrm{I}^{2}<50 \%\right)$; and (5) had no evidence of small-study effects or excess significance bias. We classified the credibility into: convincing [class I; fulfilling (1) to (5)], highly suggestive [class II; fulfilling (1) to (3)], suggestive [class III; fulfilling (1) to (2)], and weak [class IV; fulfilling only (1)].

\section{RESULTS}

Characteristics of included systematic reviews and meta-analyses

Of 339 citations, we retrieved 20 full-text articles after removing duplicates and screening at the titles and abstracts level. We excluded 328 articles for not focusing on IBS, without systematic review design, with the number of RCTs less than 10 , without treatments or outcomes of interest, without fulltext copies, without necessary data, or outdated versions. We finally included 11 systematic reviews for a total of 40 metaanalyses (330 RCTs, 86,459 participants) (Fig. 1 and Table I) [12-16, 28-33]. The meta-analyses evaluated 10 pharmacologic treatment categories [5-HT3 antagonists, 5-HT4 agonists, 5-HT3 antagonists plus 5-HT4 agonists, antidepressants, antispasmodics, antibiotics, bulking agents, guanylate cyclase-C (GCC) agonists, probiotics, and prokinetics] and 2 specific drugs (alosetron and tegaserod). The meta-analyses evaluated 11 outcomes: relief of global IBS symptoms (11 meta-analyses), responder rate (8 meta-analyses), abdominal pain (5 meta-analyses), adverse event ( 8 meta-analyses), and other outcomes. Ten (25\%) out of the 40 meta-analyses showed a small degree of heterogeneity, $6(15 \%)$ a moderate degree of heterogeneity, 19 (47.5\%) large heterogeneity and 5 (12.5\%) significantly large heterogeneity.

\section{Quality assessment}

Ten $(90.9 \%)$ systematic reviews included the components of participants, intervention, comparison, and outcomes (PICO) in the research questions and inclusion criteria. Two (18.2\%) systematic reviews stated that the review methods were established prior to the conduct of the reviews. Five (45.5\%) systematic reviews explained their selection of the study designs. Eight (72.7\%) systematic reviews used comprehensive search strategy. Study selection was performed by 2 independent reviewers in $8(72.7 \%)$ systematic reviews. Six (54.5\%) systematic reviews reported performing data extraction in duplicate. Lists of excluded studies and their justification were provided in 2 (18.2\%) systematic reviews. Ten $(90.9 \%)$ systematic reviews described adequate detail of the included studies. Ten (90.9\%) systematic reviews adopted a satisfactory technique for assessing risk of bias in each primary study; and $8(72.7 \%)$ systematic reviews assessed the potential impact of risk of bias in primary studies on the results of the meta-analyses; but only 4 (36.4\%) accounted for risk of bias in individual studies when the review authors interpreted the results. The source of funding was reported in 10 (90.9\%) systematic reviews.

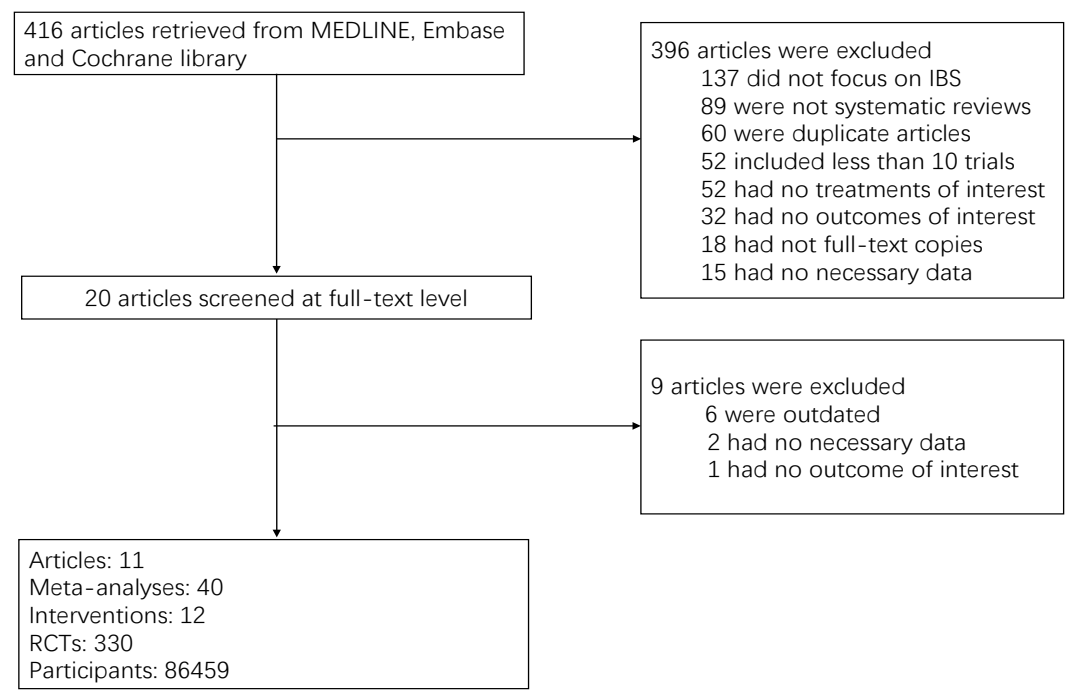

Fig. 1. Summary of study retrieval and identification. IBS: irritable bowel syndrome; RCT: randomized controlled trial. 


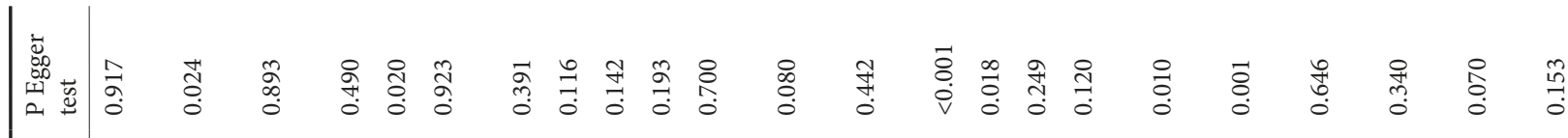

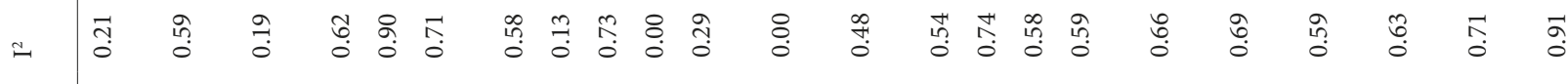

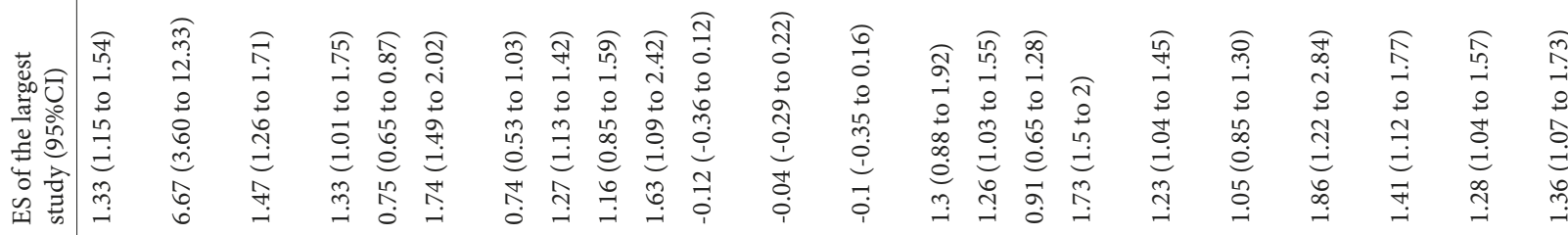

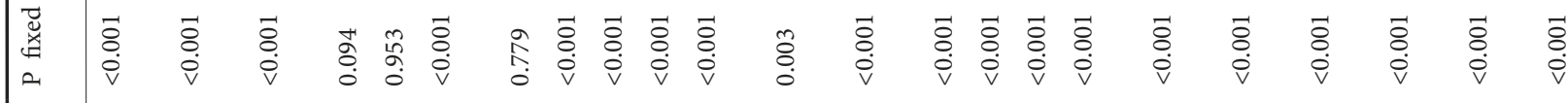

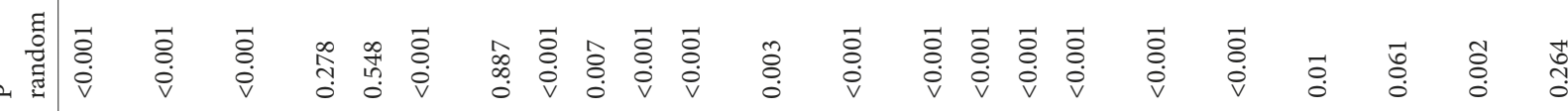

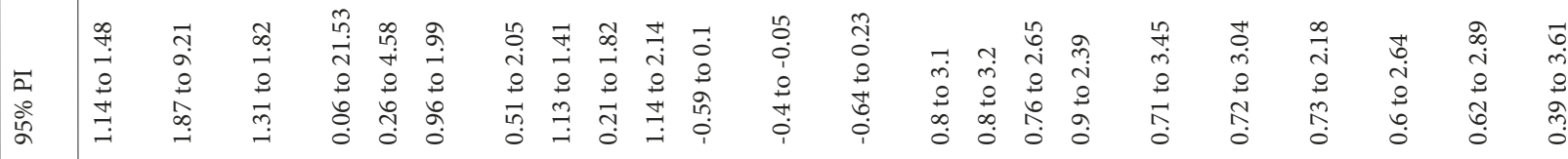

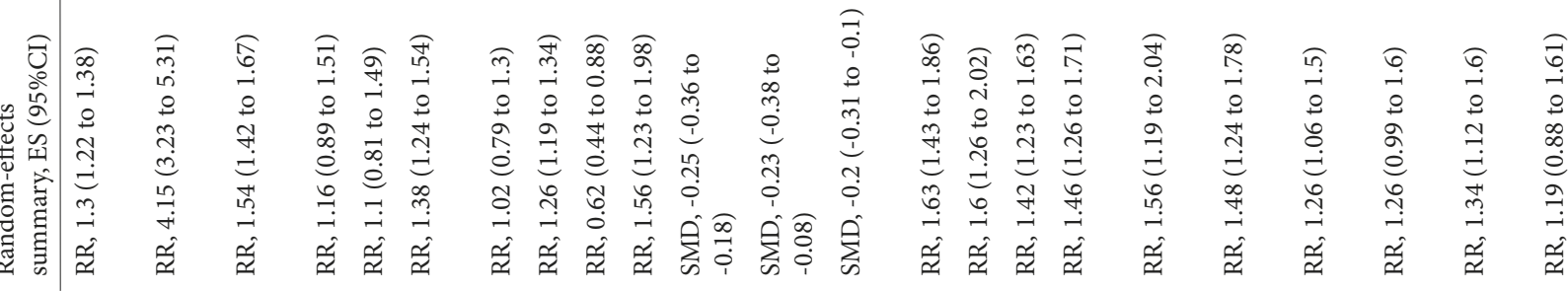

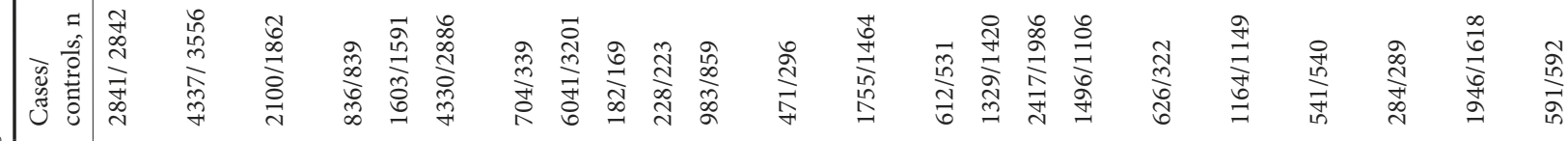
$=$ $\ddot{+}$ $n+\exists=\wedge$ \&

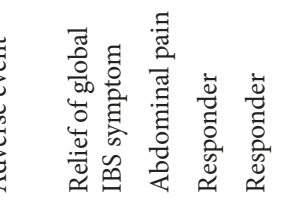

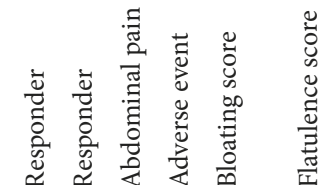

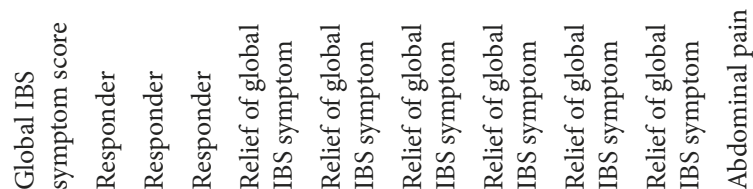

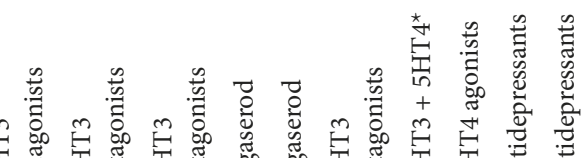

\section{(1) क क}

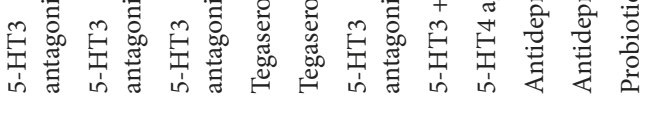

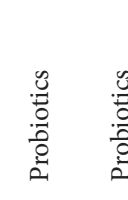

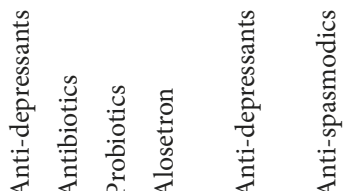

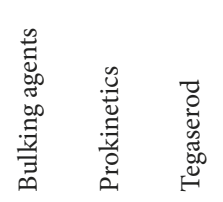

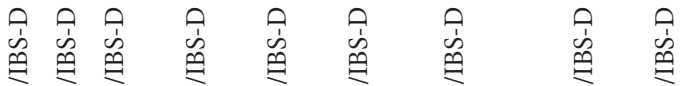

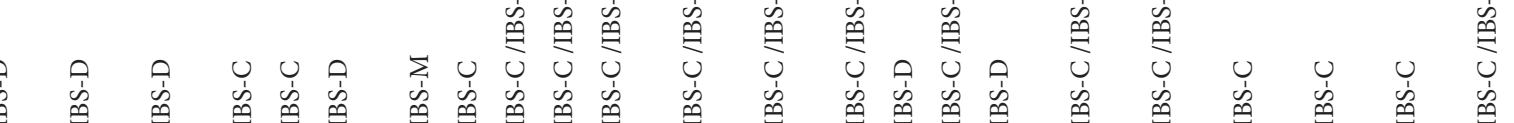

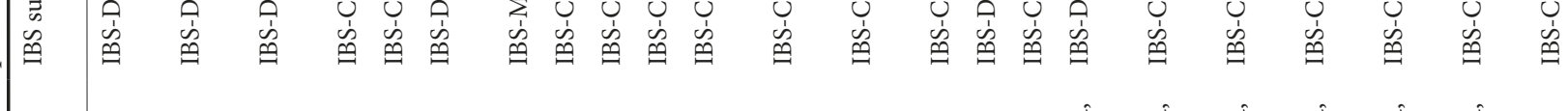




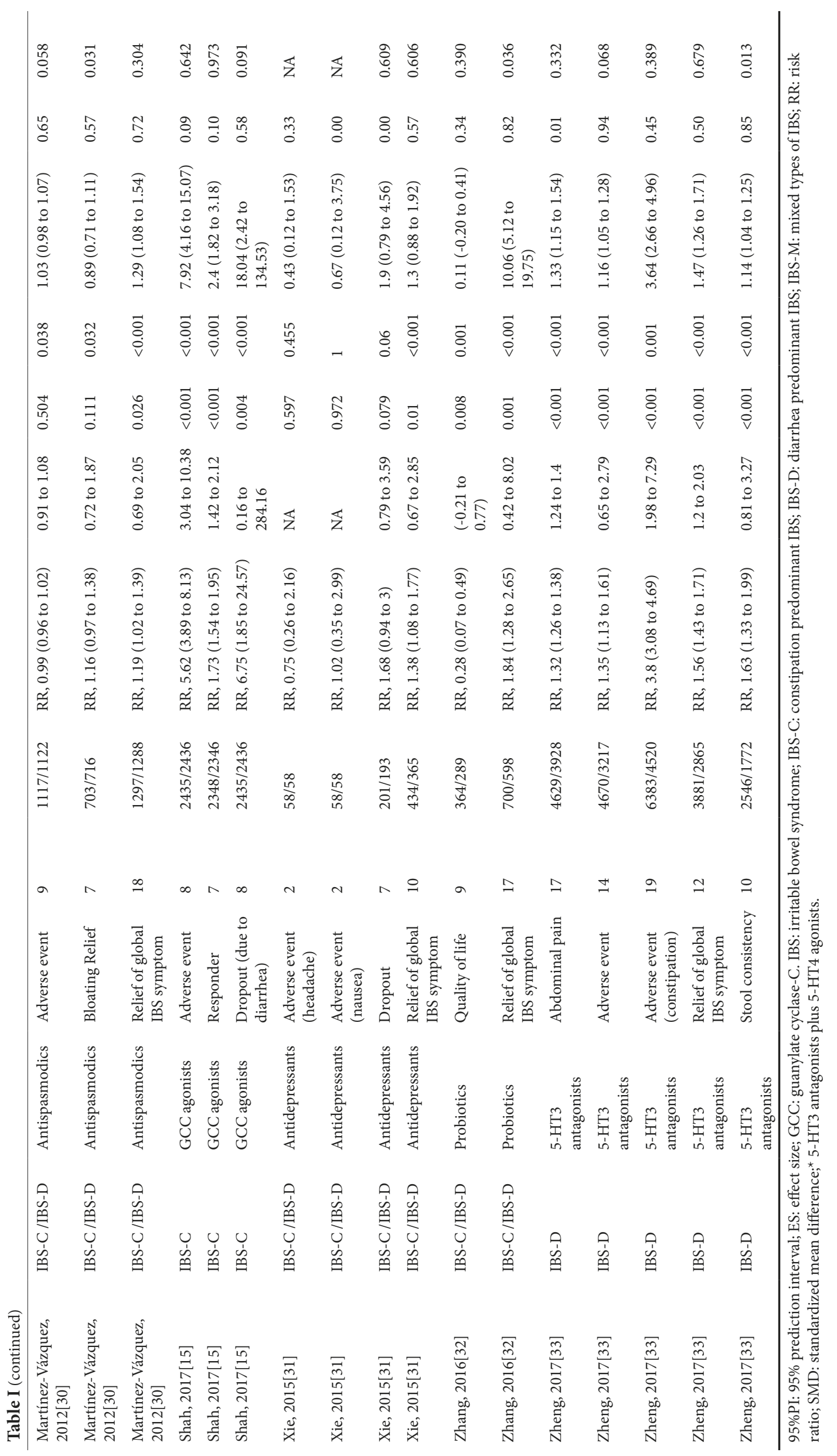


All the systematic reviews used appropriate methods in data synthesis. Eight (72.7\%) systematic reviews presented satisfactory explanation for heterogeneity observed in each meta-analysis. Six (54.5\%) systematic reviews reported investigation of publication bias and discussion of the impact of it on the results. Eight (72.7\%) systematic reviews reported potential sources of conflict of interest. The overall quality of the included systematic reviews was presented visually in Supplement file Fig 1.

We further summarized the risk-of-bias assessments in the systematic reviews (Supplementary file D chapter), and we found that 5 systematic reviews used the Cochrane riskof-bias tool, one used the revised Cochrane tool, four used the Jadad scale, and one used a 5-point scale. The systematic reviews assessed 5-HT3 antagonists had a low risk of bias in randomization methods-random sequence generation and allocation concealment $[16,28,33]$. The systematic review assessing antispasmodics also had a low risk of bias in randomization methods [30], but the systematic review assessing GCC had $40 \%$ of the studies with an unclear risk of bias in randomization methods [15]. The systematic reviews assessing prebiotics, synbiotics, and probiotics $[13,32]$ and the systematic reviews assessing antidepressants had similar findings [12, 31].

\section{Relief of global IBS symptoms}

Eight meta-analyses (86 RCTs, 19,397 participants) assessed the relief of global IBS symptoms in 6 treatment categories and 2 drugs (Fig. 2 and Table II). The results showed that 5-HT3 antagonists, antidepressants, antispasmodics, bulking agents, probiotics, alosetron and tegaserod were significantly superior over placebo. None of the treatments had level I evidence; 5-HT3 antagonists, antispasmodics, and alosetron were with level II evidence. Probiotics showed the largest effect size ( $\mathrm{RR}=1.84,95 \% \mathrm{CI}: 1.28-2.65$, level III evidence).

\section{Abdominal pain}

Four meta-analyses (32 RCTs, 11,766 participants) assessed abdominal pain in 3 treatment categories and one drug (Fig. 2 and Table II); only 5-HT3 antagonists showed superiority over placebo (level I evidence), and it had the largest effect size in relieving abdominal pain ( $\mathrm{RR}=1.32$, 95\%CI: 1.26-1.38).

\section{Responder rate}

Eight meta-analyses (105 RCTs, 33,684 participants) assessed responder rate in 7 treatment categories and one drug (Fig. 2 and Table II). 5-HT3 antagonists, 5-HT4 agonists, antidepressants, antibiotics, GCC agonists, and probiotics showed significant superiority over placebo. GCC agonists

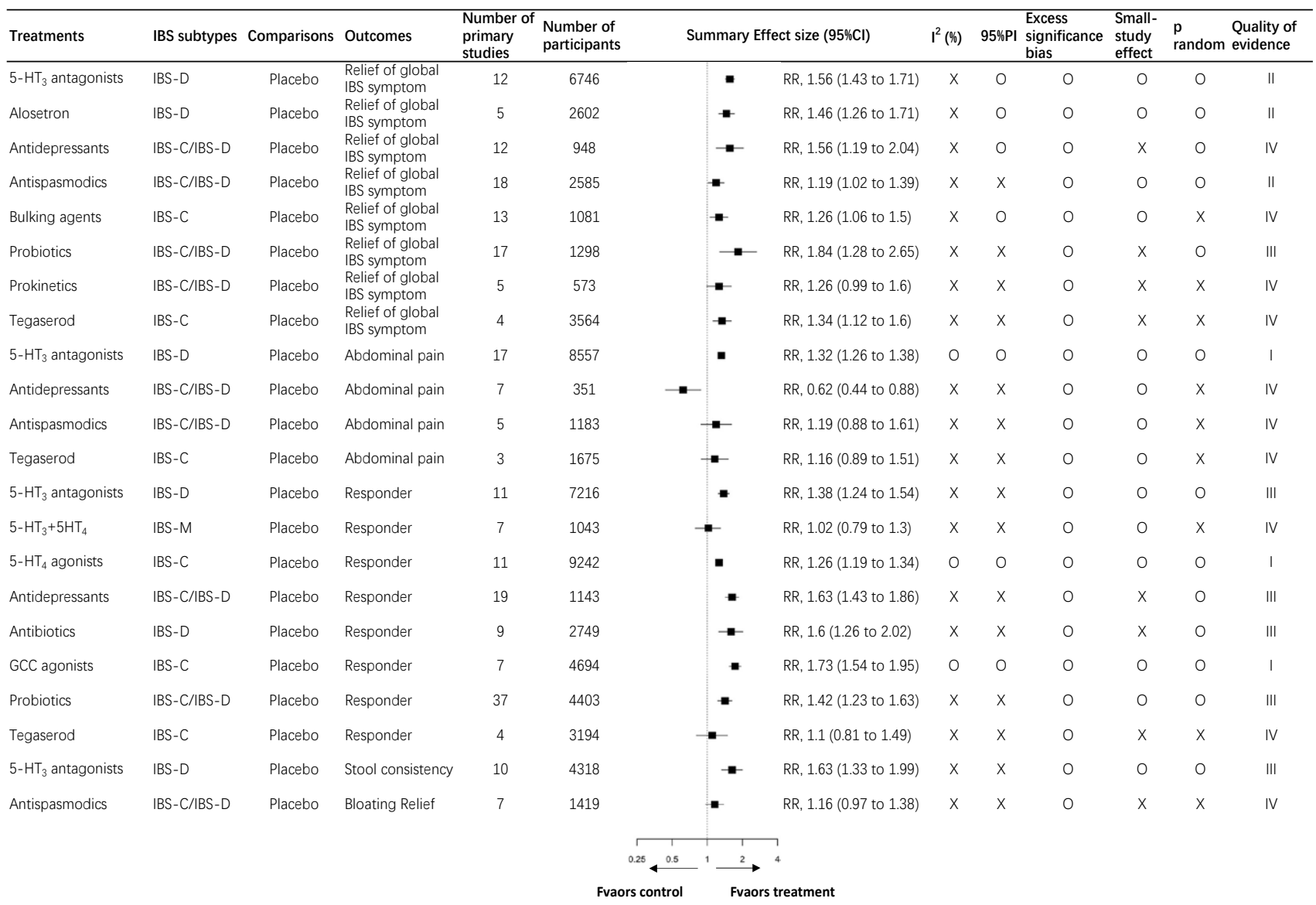

Fig. 2. Effect of pharmacologic treatment on efficacy outcomes.

Abbrev.: 95\%CI: 95\% confidence interval. 95\%PI: 95\% prediction interval. IBS: irritable bowel syndrome. IBS-C: constipation predominant IBS. IBS-D: diarrhea predominant IBS; IBS-M: mixed types of IBS. P random: p-values that were calculated by using random-effects model. RR: relative ratio. X: violated the criteria; O: fulfilled the criteria; I: convincing evidence. II: highly suggestive. III: suggestive evidence: IV: weak evidence. Footnotes: The credibility of current evidenceand the credibility classification are detailed in Methods. 
had the largest effect size (RR=1.73, 95\%CI: 1.54-1.95]) and was classified as level I evidence; the effect of 5-HT4 agonists on the responder rate was also classified at level I evidence $(\mathrm{RR}=1.26,95 \% \mathrm{CI}: 1.19-1.34)$.

\section{Other efficacy outcomes}

One meta-analysis (10 RCTs, 4,318 participants) assessed the effect of 5-HT3 antagonists on stool frequency; the result showed that it was significantly superior over placebo

Table II. Observed and expected number of studies with positive findings in the eligible meta-analyses

\begin{tabular}{|c|c|c|c|c|c|c|c|c|}
\hline Treatments & IBS subtypes & Comparisons & Outcomes & $\begin{array}{l}\text { Studies, } \\
\mathrm{n}\end{array}$ & $\begin{array}{l}\text { Expected } \\
\text { number (E) }\end{array}$ & $\begin{array}{l}\text { Observed } \\
\text { number }(\mathrm{O})\end{array}$ & $\mathrm{O} / \mathrm{E}$ ratio & $\overline{p \text {-value }}$ \\
\hline 5-HT3 antagonists & IBS-D & Placebo & $\begin{array}{l}\text { Relief of global IBS } \\
\text { symptom }\end{array}$ & 12 & 7.03 & 9 & 1.28 & 0.953 \\
\hline Alosetron & IBS-D & Placebo & $\begin{array}{l}\text { Relief of global IBS } \\
\text { symptom }\end{array}$ & 5 & 4.12 & 3 & 0.73 & 1 \\
\hline Antidepressants & IBS-C /IBS-D & Placebo & $\begin{array}{l}\text { Relief of global IBS } \\
\text { symptom }\end{array}$ & 12 & 2.63 & 5 & 1.90 & 0.739 \\
\hline Antispasmodics & IBS-C /IBS-D & Placebo & $\begin{array}{l}\text { Relief of global IBS } \\
\text { symptom }\end{array}$ & 18 & 7.63 & 8 & 1.05 & 1 \\
\hline Bulking agents & IBS-C & Placebo & $\begin{array}{l}\text { Relief of global IBS } \\
\text { symptom }\end{array}$ & 13 & 9.58 & 6 & 0.63 & 0.69 \\
\hline Probiotics & IBS-C /IBS-D & Placebo & $\begin{array}{l}\text { Relief of global IBS } \\
\text { symptom }\end{array}$ & 17 & 7.34 & 11 & 1.50 & 0.689 \\
\hline Prokinetics & IBS-C & Placebo & $\begin{array}{l}\text { Relief of global IBS } \\
\text { symptom }\end{array}$ & 5 & 2.08 & 2 & 0.96 & 1 \\
\hline Tegaserod & IBS-C & Placebo & $\begin{array}{l}\text { Relief of global IBS } \\
\text { symptom }\end{array}$ & 4 & 1.2 & 4 & 3.33 & 0.688 \\
\hline 5-HT3 antagonists & IBS-D & Placebo & Abdominal pain & 17 & 8.6 & 12 & 1.40 & 0.754 \\
\hline Antidepressants & IBS-C /IBS-D & Placebo & Abdominal pain & 7 & 2.1 & 4 & 1.90 & 0.859 \\
\hline Antispasmodics & IBS-C /IBS-D & Placebo & Abdominal pain & 5 & 3.43 & 2 & 0.58 & 1 \\
\hline Tegaserod & IBS-C & Placebo & Abdominal pain & 3 & 0.9 & 1 & 1.11 & 1 \\
\hline 5-HT3 antagonists & IBS-D & Placebo & Responder & 11 & 4.7 & 8 & 1.70 & 0.699 \\
\hline 5-HT4 agonists & IBS-C & Placebo & Responder & 11 & 5.98 & 6 & 1.00 & 1 \\
\hline 5-HT3+5-HT4 & IBS-M & Placebo & Responder & 7 & 4.06 & 1 & 0.25 & 0.512 \\
\hline Antidepressants & IBS-C /IBS-D & Placebo & Responder & 19 & 7.85 & 9 & 1.15 & 1 \\
\hline Antibiotics & IBS-D & Placebo & Responder & 9 & 4.31 & 6 & 1.39 & 0.978 \\
\hline GCC agonists & IBS-C & Placebo & Responder & 7 & 6.79 & 7 & 1.03 & 1 \\
\hline Probiotics & IBS-C /IBS-D & Placebo & Responder & 37 & 17.44 & 13 & 0.75 & 0.643 \\
\hline Tegaserod & IBS-C & Placebo & Responder & 4 & 3.35 & 2 & 0.60 & 1 \\
\hline 5-HT3 antagonists & IBS-D & Placebo & Stool consistency & 10 & 8.06 & 8 & 0.99 & 1 \\
\hline Antispasmodics & IBS-C /IBS-D & Placebo & Bloating Relief & 7 & 2.86 & 3 & 1.05 & 1 \\
\hline 5-HT3 antagonists & IBS-D & $\begin{array}{l}\text { Placebo or } \\
\text { active control }\end{array}$ & Adverse event & 14 & 13 & 14 & 1.08 & 1 \\
\hline 5-HT3 antagonists & IBS-D & Placebo & $\begin{array}{l}\text { Adverse event } \\
\text { (constipation) }\end{array}$ & 19 & 14.53 & 16 & 1.10 & 1 \\
\hline Antidepressants & IBS-C /IBS-D & Placebo & Adverse event & 8 & 4.42 & 2 & 0.45 & 0.732 \\
\hline Antispasmodics & IBS-C /IBS-D & Placebo & Adverse event & 9 & 4.22 & 0 & 0.00 & 0.183 \\
\hline GCC agonists & IBS-C & Placebo & Adverse event & 8 & 5.03 & 7 & 1.39 & 0.962 \\
\hline Antidepressants & IBS-C /IBS-D & Placebo & Dropout & 7 & 1.02 & 0 & 0.00 & 1 \\
\hline GCC agonists & IBS-C & Placebo & $\begin{array}{l}\text { Dropout (due to } \\
\text { diarrhea) }\end{array}$ & 8 & 4.94 & 2 & 0.40 & 0.63 \\
\hline Probiotics & IBS-C /IBS-D & Placebo & Bloating score & 26 & 1.49 & 1 & 0.67 & 1 \\
\hline Probiotics & IBS-C /IBS-D & Placebo & Flatulence score & 11 & 0.58 & 1 & 1.72 & 1 \\
\hline Probiotics & IBS-C /IBS-D & Placebo & $\begin{array}{l}\text { Global IBS } \\
\text { symptom score }\end{array}$ & 35 & 3.15 & 6 & 1.90 & 0.592 \\
\hline Probiotics & IBS-C /IBS-D & Placebo & Quality of life & 9 & 1.02 & 4 & 3.92 & 0.498 \\
\hline
\end{tabular}

Abbrev.: O/E: the observed number of trials with positive findings versus the expected number; IBS: irritable bowel syndrome; IBS-C: constipation predominant IBS; IBS-D: diarrhea predominant IBS; IBS-M: mixed types of IBS. 
( $\mathrm{RR}=1.63,95 \% \mathrm{CI}: 1.33-1.99$, level III evidence). One metaanalysis (7 RCTs, 1,419 participants) assessed the effect of antispasmodics on the relief of bloating; the result showed no positive findings (level IV evidence) (Fig. 2 and Table II). Three meta-analyses (72 RCTs, 5,828 participants) assessed the effect of probiotics on the bloating score, flatulence score, and global IBS symptom score, respectively (Supplement file Fig. 1).

The results showed that probiotics were significantly superior over placebo for all the three outcomes (bloating score: $\mathrm{SMD}=-0.25,95 \% \mathrm{CI}:-0.36--0.18$, level III evidence; flatulence score: $\mathrm{SMD}=-0.23,95 \% \mathrm{CI}:-0.38--0.08$, level III evidence; global IBS symptom score: $\mathrm{SMD}=-0.2,95 \% \mathrm{CI}$ : $-0.31--0.1$, level IV evidence).

\section{Adverse event and dropout}

Four meta-analyses (39 RCTs, 15,448 participants) assessed the overall adverse event rate in 4 treatment categories. 5-HT3 antagonists ( $\mathrm{RR}=1.35,95 \% \mathrm{CI}: 1.13-1.61$, level III evidence), antidepressants ( $\mathrm{RR}=1.56,95 \% \mathrm{CI}: 1.23-1.98$, level IV evidence), and GCC agonists ( $\mathrm{RR}=5.62$, 95\%CI: 3.89-8.13, level I evidence) had significantly higher adverse event rate than placebo. Three meta-analyses analysis assessed a specific adverse event; one (19 RCTs, 10,903 participants) assessed constipation in 5-HT3 antagonists and showed significant more events of constipation in 5-HT3 antagonists than placebo $(\mathrm{RR}=3.8$, 95\% CI: 3.08-4.69, level I evidence); another two meta-analyses assessed the rate of headache or nausea after administration of antidepressants and found no significant difference between antidepressants and placebo. Two metaanalysis assessed the rate of dropout due to adverse events; one (8 RCTs, 4,871 participants) found a higher dropout rate in GCC agonists than placebo $(\mathrm{RR}=6.75,95 \% \mathrm{CI}$ : $1.85-24.57$, level IV evidence). Fig. 3 and Table II show the credibility of current evidence on adverse events and dropouts after using pharmacologic treatments.

\section{DISCUSSION}

Our umbrella review summarized current evidence on pharmacologic treatments in the management of IBS. We had several findings on the basis of statistical assessment: (1) most of the meta-analyses reported significant superiority of pharmacologic treatment over placebo; (2) the evidence for 5-HT3 antagonists, antispasmodics, and alosetron were highly suggestive for relieving global IBS symptoms; (3) 5-HT4 agonists and GCC agonists offered convincing evidence for improving the responder rate in the management of IBS; (4) 5-HT3 antagonists offered convincing evidence for relieving abdominal pain in patients with IBS; (5) there was convincing evidence that 5-HT3 caused more constipation events and GCC agonists caused more overall adverse events.

We also report several findings from the quality evaluation on the basis of AMSTAR 2 assessment: (1) most of the included systematic reviews were unregistered prior to their conduction; (2) most of the systematic reviews did not discuss the impact of risk of bias in individual studies when interpreting the results. Although risk-of-bias assessment is suggested, many systematic reviews still assessed the quality of an RCT on the basis of rating scales such as the Jadad scale which gives a general score for the quality but misses many domains of the risk of bias (such selective outcome reporting). More than one third of our included SR adopted the Jadad scale, which indicates a lack of assessment of risk of bias in domains other than randomization methods and blinding. Risk of bias in randomization methods (random sequence generation and allocation concealment) was still a problem, as shown in our review. Our review showed that RCTs assessing probiotics, antibiotics, and anti-depressants had an unclear risk-of-bias in randomization methods and the result indicates that future RCTs should strictly follow the CONSORT statement.

The first strength of the review was that we used an umbrella review approach. Compared with overviews of systematic

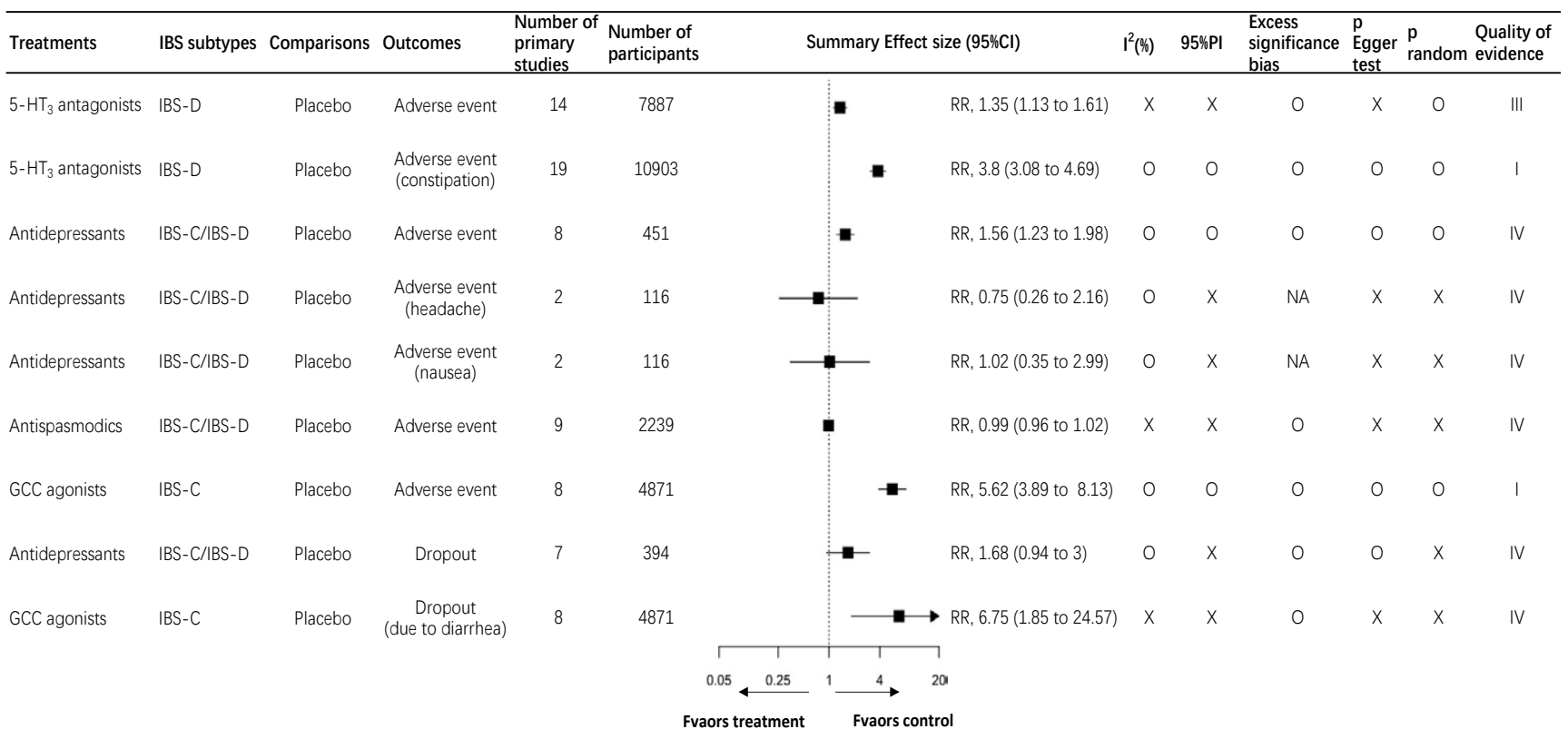

Fig. 3. Effect of pharmacologic treatments on safety outcomes. (See the Abbreviations and Footnotes of Fig. 2) 
reviews, the umbrella review added quantitative assessment of the outcomes and adopted standard criteria to classified evidence into different levels of confidence [22]. To the best of our knowledge, this is the first umbrella review that has quantitatively and comprehensively summarized currently available evidence of pharmacologic treatments for patients with IBS. The second strength was the provided map of the strength and credibility of current evidence on pharmacologic treatments for IBS. The evidence map reveals the knowledge gap in the effectiveness and safety of the pharmacologic treatments and provides insights for future studies. The third strength was that it provided an overview of the effect sizes of the treatments, which facilitates decision making for clinical practitioners and patients with IBS. The fourth strength was that we reported each methodological evaluation items separately instead of reporting a general score, which helps investigators to avoid methodological deficits when designing future meta-analyses.

We noted that $80 \%$ of the meta-analyses had statistically significant heterogeneity, and most of them had $\mathrm{I}^{2}>50 \%$. The amount of heterogeneity was larger than expected, since we assessed meta-analyses that included only RCTs; meta-analyses that included observation studies were excluded in the review. The large heterogeneity reduced the credibility of treatments with promising effect sizes, eg, probiotics in relieving global IBS symptoms $(\mathrm{RR}=1.84)$. The large heterogeneity may partly be explained by different treatments in the same category which might have heterogeneous treatment effect sizes [34]. However, we found that meta-analyses examining alosetron and tegaserod also showed significant heterogeneity, which indicates that the study population and the outcome measurements may also be the source of heterogeneity. Regarding the study population, IBS was subdivided into four subtypes: diarrheapredominant IBS, constipation-predominant IBS, mixed IBS, and unspecified IBS [4]. Although these four subtypes shared a common pathological base of IBS, they had their own features in treatments, eg, alosetron was usually selected for women with diarrhea-predominant IBS [35]. Treatments like probiotics that have no clear indications for a specific subtype may exert a heterogeneous effect on different IBS subtypes, which may be one of the causes for significant heterogeneity. Regarding the outcome measurements, a previous study showed that disagreement was found between patient-reported outcomes and clinician-reported outcomes in the assessment of IBS [36], and another study showed that patient-reported outcomes in IBS were influenced by psychological and somatic symptoms [37].

We found that more than $2 / 3$ of the meta-analyses had a $95 \%$ PI containing null value, and it downgraded the credibility of the evidence. The 95\%PI in a meta-analysis addresses the actual dispersion of true effect sizes, and shows that the true effect of a treatment in $95 \%$ of new studies will fall into the range of $95 \%$ PI [38]. The 95\%PI is usually influenced by the standard deviation of true effect sizes (tau square) or the number of studies. The first explanation for most of the included meta-analyses having a 95\%PI containing null value is the large heterogeneity, which indicates a large value of tau square and therefore leads to a wider 95\%PI. The second explanation is that the number of original studies is insufficient; half of the included meta-analyses had a number of RCTs less than 10 . The width of the $95 \%$ PI drops sharply as the number of studies increases from 6 to 24 .

Although we found no evidence of an excess of significance bias in the review, we still found suspicious signs of the bias through the $\mathrm{O} / \mathrm{E}$ ratios. In assessing the effect of tegaserod on relief of global IBS symptoms, we found an O/E ratio as large as 3.33; and we also found $\mathrm{O} / \mathrm{E}$ ratios larger than 1 in several pharmacologic treatments, including tegaserod, 5-HT3 antagonists, and antidepressants for relieving abdominal pain, antispasmodics, 5-HT3 antagonists, probiotics, antidepressants, and tegaserod for relieving global IBS symptoms, and GCC agonists, antidepressants, antibiotics, and 5-HT3 antagonists for improving the responder rate. These findings suggested that excess significance bias was prevalent in pharmacologic trials for IBS. The first explanation may be that studies with positive findings are easier to get published in time while those with negative findings usually remain unpublished or in a delayed publishing process (time lag) and the Egger's test is not sensitive in detecting this publication bias when a meta-analysis includes a number of RCTs lower than 20 [39]. The second explanation is the large number of small sample size RCTs in each metaanalysis, especially in RCTs of antidepressants [40], probiotics [13], antispasmodics [14], and bulking agents [14]. There is no standard for defining a high risk of significance bias, and we tend to define that a $\mathrm{O} / \mathrm{E}$ ratio over 3 should be considered $[39,41]$. According to the definition, the results of probiotics (3.92) and tegaserod (3.33) should be interpreted with caution.

We found that most of the included systematic reviews assessed pharmacological treatments as monotherapy; except in Ford's review [29], the effect of 5-HT3 antagonists plus 5-HT4 agonists was assessed. IBS is a disease with a complex pathological mechanism. In routine practice, complex interventions (combining two or more pharmacological treatments with non-pharmacological treatment) are commonly used. The reason (why few systematic reviews have been conducted in complex interventions for IBS) could be that original studies are sparse and the regimens of complex interventions are diverse. Diversity in treatment regimens could be solved by a component network meta-analysis [42], but the lack of original studies requires scholars to conduct more pragmatic studies that adopt regimens used in routine practice.

Our umbrella review had several limitations. First, owing to the inclusion of a small number of RCTs in the meta-analysis, the tests of publication bias and excess significance bias did not reveal the actual fact, so the results of them should be cautiously interpreted. Second, we searched for systematic reviews that included at least 10 RCTs, which may miss some systematic reviews on the topic. However, a previously published umbrella review adopted the method to avoid the inclusion of low-quality evidence and to avoid the introduction of meta-analyses without a comprehensive search for RCTs [24]. Third, the umbrella review provided an overview of systematic reviews instead of RCTs, which leads to a consequence that the included references were not up-to-date. In addition, it will miss some of the interventions with relatively few RCTs, or it will categorize these interventions into a large group (eg, rifaximin was grouped into antibiotics in our umbrella review). 


\section{CONCLUSIONS}

Our umbrella review found suggestive evidences for 5-HT3 antagonists, 5-HT4 agonists and GCC agonists, antispasmodics, and alosetron for the treatment of IBS. 5-HT3 antagonists and GCC agonists were showed via convincing evidence to have more adverse events. However, due to a potential risk of excess significance bias, publication bias and individual study biases, the results should be interpreted with caution.

Conflicts of interest: None to declare.

Authors' contributions: M.C. and H.Z conceived and designed the study, had full access to all data in this study, took responsibility for the integrity of the data and the accuracy of the data analysis. T.-C.T, D.Q., L.Y. data analysis and interpretation. H.Z: statistical analysis. M.C: drafted the manuscript. All authors critically revised the manuscript for important intellectual content.

Acknowledgements: M.C. and H.Z. received grants (no. 81774321 and no. 81473777) from the National Natural Science Foundation of China. The funders had no role in the design and conduct of the study, and it had no role in the decision process to submit the manuscript for publication.

Supplementary material: To access the supplementary material visit the online version of the J Gastrointestin Liver Dis at http://dx.doi. org/10.15403/jgld-817

\section{REFERENCES}

1. Sperber AD, Dumitrascu D, Fukudo S, et al. The global prevalence of IBS in adults remains elusive due to the heterogeneity of studies: a Rome Foundation working team literature review. Gut 2017;66:1075-1082. doi:10.1136/gutjnl-2015-311240

2. Lovell RM, Ford AC. Global prevalence of and risk factors for irritable bowel syndrome: a meta-analysis. Clin Gastroenterol Hepatol 2012;10:712-721.e4. doi:10.1016/j.cgh.2012.02.029

3. Dean BB, Aguilar D, Barghout V, et al. Impairment in work productivity and health-related quality of life in patients with IBS. Am J Manag Care 2005;11(1 Suppl):S17-S26.

4. Ford AC, Lacy BE, Talley NJ. Irritable Bowel Syndrome. N Engl J Med 2017;376:2566-2578. doi:10.1056/NEJMra1607547

5. Everhart JE, Ruhl CE. Burden of digestive diseases in the United States part I: overall and upper gastrointestinal diseases. Gastroenterology 2009;136:376-386. doi:10.1053/j.gastro.2008.12.015

6. Flacco ME, Manzoli L, De Giorgio R, et al. Costs of irritable bowel syndrome in European countries with universal healthcare coverage: a meta-analysis. Eur Rev Med Pharmacol Sci 2019;23:2986-3000. doi:10.26355/eurrev_201904_17580

7. McKenzie YA, Bowyer RK, Leach H, et al. British Dietetic Association systematic review and evidence-based practice guidelines for the dietary management of irritable bowel syndrome in adults (2016 update). J Hum Nutr Diet 2016;29:549-575. doi:10.1111/jhn.12385

8. Moayyedi P, Andrews CN, MacQueen G, et al. Canadian Association of Gastroenterology Clinical Practice Guideline for the Management of Irritable Bowel Syndrome (IBS). J Can Assoc Gastroenterol 2019;2:6-29. doi:10.1093/jcag/gwy071
9. Ervin CM, Mangel AW. Clinical trials in irritable bowel syndrome: a review. Rev Recent Clin Trials 2013;8:9-22. doi:10.2174/15748871113 08010003

10. Ford AC, Brandt LJ, Young C, Chey WD, Foxx-Orenstein AE, Moayyedi P. Efficacy of 5-HT3 antagonists and 5-HT4 agonists in irritable bowel syndrome: systematic review and meta-analysis. Am J Gastroenterol 2009;104:1831-1843.

11. Andresen V, Montori VM, Keller J, West CP, Layer P, Camilleri M. Effects of 5-hydroxytryptamine (serotonin) type 3 antagonists on symptom relief and constipation in nonconstipated irritable bowel syndrome: a systematic review and meta-analysis of randomized controlled trials. Clin Gastroenterol Hepatol 2008;6:545-555. doi:10.1016/j. cgh.2007.12.015

12. Ford AC, Lacy BE, Harris LA, Quigley EMM, Moayyedi P. Effect of Antidepressants and Psychological Therapies in Irritable Bowel Syndrome: An Updated Systematic Review and Meta-Analysis. Am J Gastroenterol 2019;114:21-39. doi:10.1038/s41395-018-0222-5

13. Ford AC, Harris LA, Lacy BE, Quigley EMM, Moayyedi P. Systematic review with meta-analysis: the efficacy of prebiotics, probiotics, synbiotics and antibiotics in irritable bowel syndrome. Aliment Pharmacol Ther 2018;48:1044-1060. doi:10.1111/apt.15001

14. Lesbros-Pantoflickova D, Michetti P, Fried M, Beglinger C, Blum AL. Meta-analysis: The treatment of irritable bowel syndrome. Aliment Pharmacol Ther 2004;20:1253-1269. doi:10.1111/j.13652036.2004.02267.x

15. Shah ED, Kim HM, Schoenfeld P. Efficacy and Tolerability of Guanylate Cyclase-C Agonists for Irritable Bowel Syndrome with Constipation and Chronic Idiopathic Constipation: A Systematic Review and Meta-Analysis. Am J Gastroenterol 2018;113:329-338. doi:10.1038/ ajg.2017.495

16. Evans BW, Clark WK, Moore DJ, Whorwell PJ. Tegaserod for the treatment of irritable bowel syndrome and chronic constipation. Cochrane Database Syst Rev 2007;(4):CD003960. doi:10.1002/14651858. CD003960.pub3

17. Ioannidis J. Next-generation systematic reviews: prospective metaanalysis, individual-level data, networks and umbrella reviews. Br J Sports Med 2017;51:1456-1458. doi:10.1136/bjsports-2017-097621

18. Neuenschwander M, Ballon A, Weber KS, et al. Role of diet in type 2 diabetes incidence: umbrella review of meta-analyses of prospective observational studies. BMJ 2019;366:12368. doi: 10.1136/bmj.12368

19. Piovani D, Danese S, Peyrin-Biroulet L, Nikolopoulos GK, Lytras T, Bonovas. S. Environmental Risk Factors for Inflammatory Bowel Diseases: an Umbrella Review of Meta-analyses. Gastroenterology 2019;157:647-659.e4. doi:10.1053/j.gastro.2019.04.016

20. Khan SU, Khan MU, Riaz H, et al. Effects of Nutritional Supplements and Dietary Interventions on Cardiovascular Outcomes: An Umbrella Review and Evidence Map. Ann Intern Med 2019;171:190-198. doi:10.7326/M19-0341

21. Hutton B, Salanti G, Caldwell DM, et al. The PRISMA extension statement for reporting of systematic reviews incorporating network meta-analyses of health care interventions: checklist and explanations. Ann Intern Med 2015;162:777-784.

22. Aromataris E, Fernandez R, Godfrey CM, Holly C, Khalil H, Tungpunkom P. Summarizing systematic reviews: methodological development, conduct and reporting of an umbrella review approach. Int J Evid Based Healthc 2015;13:132-140. doi:10.1097/ XEB.0000000000000055

23. Qin D, Yue L, Xue B, Chen M, Tang TC, Zheng H. Pharmacological treatments for patients with irritable bowel syndrome: An umbrella 
review of systematic reviews and meta-analyses. Medicine (Baltimore) 2019;98:e15920. doi:10.1097/MD.0000000000015920

24. Dragioti E, Karathanos V, Gerdle B, Evangelou E. Does psychotherapy work? An umbrella review of meta-analyses of randomized controlled trials. Acta Psychiatr Scand 2017;136:236-246. doi:10.1111/ acps. 12713

25. Shea BJ, Reeves BC, Wells G, et al. AMSTAR 2: a critical appraisal tool for systematic reviews that include randomised or non-randomised studies of healthcare interventions, or both. BMJ 2017;358:j4008. doi:10.1136/ bmj.j4008

26. Ioannidis JP, Trikalinos TA. An exploratory test for an excess of significant findings. Clin Trials. 2007;4:245-253. doi:10.1177/1740774507079441

27. Belbasis L, Bellou V, Evangelou E, Ioannidis JPA, Tzoulaki I. Environmental risk factors and multiple sclerosis: an umbrella review of systematic reviews and meta-analyses. Lancet Neurol 2015;14:263-273. doi: 10.1016/S1474-4422(14)70267-4

28. Andresen V, Montori VM, Keller J, West CP, Layer P, Camilleri M. Effects of 5-hydroxytryptamine (serotonin) type 3 antagonists on symptom relief and constipation in nonconstipated irritable bowel syndrome: a systematic review and meta-analysis of randomized controlled trials. Clin Gastroenterol Hepatol 2008;6:545-555. doi:10.1016/j.cgh.2007.12.015

29. Ford AC, Brandt LJ, Young C, Chey WD, Foxx-Orenstein AE, Moayyedi P. Efficacy of 5-HT3 antagonists and 5-HT4 agonists in irritable bowel syndrome: systematic review and meta-analysis. Am J Gastroenterol 2009;104:1831-1843.

30. Martínez-Vázquez MA, Vázquez-Elizondo G, González-González JA, Gutiérrez-Udave R, Maldonado-Garza HJ, Bosques-Padilla FJ. Effect of antispasmodic agents, alone or in combination, in the treatment of Irritable Bowel Syndrome: Systematic review and meta-analysis. Rev Gastroenterol de Mex 2012;77:82-90. doi:10.1016/j.rgmx.2012.04.002

31. Xie C, Tang Y, Wang Y, et al. Efficacy and Safety of Antidepressants for the Treatment of Irritable Bowel Syndrome: A Meta-Analysis. PLoS ONE 2015;10:e0127815. doi:10.1371/journal.pone.0127815

32. Zhang Y, Li L, Guo C, et al. Effects of probiotic type, dose and treatment duration on irritable bowel syndrome diagnosed by Rome III criteria: a meta-analysis. BMC Gastroenterol 2016;16:62. doi:10.1186/s12876016-0470-z
33. Zheng Y, Yu T, Tang Y, et al. Efficacy and safety of 5-hydroxytryptamine 3 receptor antagonists in irritable bowel syndrome: A systematic review and meta-analysis of randomized controlled trials. PLoS One 2017;12:e0172846. doi:10.1371/journal.pone.0172846

34. Fletcher J. What is heterogeneity and is it important? BMJ 2007;334:9496. doi:10.1136/bmj.39057.406644.68

35. Lacy BE, Nicandro JP, Chuang E, Earnest DL. Alosetron use in clinical practice: significant improvement in irritable bowel syndrome symptoms evaluated using the US Food and Drug Administration composite endpoint. Therap Adv Gastroenterol 2018;11:1756284818771674. doi:10.1177/1756284818771674

36. Chassany O, Le-Jeunne P, Duracinsky M, Schwalm M-S, Mathieu M. Discrepancies between patient-reported outcomes and clinicianreported outcomes in chronic venous disease, irritable bowel syndrome, and peripheral arterial occlusive disease. Value Health 2006;9:39-46. doi:10.1111/j.1524-4733.2006.00079.x

37. Lackner J, Jaccard J, Baum C, et al. Patient-reported outcomes for irritable bowel syndrome are associated with patients' severity ratings of gastrointestinal symptoms and psychological factors. Clin Gastroenterol Hepatol 2011;9:957-964.e1. doi:10.1016/j.cgh.2011.06.014

38. Lin L. Use of Prediction Intervals in Network Metaanalysis. JAMA Netw Open 2019;2:e199735. doi:10.1001/ jamanetworkopen.2019.9735

39. Ioannidis JPA. Clarifications on the application and interpretation of the test for excess significance and its extensions. J Math Psychol 2013;57:184-187. doi:10.1016/j.jmp.2013.03.002

40. Ford AC, Lacy BE, Harris LA, Quigley EMM, Moayyedi P. Effect of Antidepressants and Psychological Therapies in Irritable Bowel Syndrome: An Updated Systematic Review and Meta-analysis. Am J Gastroenterol 2019;114:21-39. doi:10.1038/s41395-018-0222-5

41. Ioannidis JP. Excess Significance Bias in the Literature on Brain Volume Abnormalities. Arch Gen Psychiatry 2011;68:773-780. doi:10.1001/ archgenpsychiatry.2011.28

42. Mills EJ, Thorlund K, Ioannidis JPA. Calculating additive treatment effects from multiple randomized trials provides useful estimates of combination therapies. J Clin Epidemiol 2012;65:1282-1288. doi:10.1016/j.jclinepi.2012.07.012 